\title{
Análise do conhecimento de moradores quanto à transmissibilidade e prevenção da tuberculose: implicações biopsicossociais
}

\author{
Analysis of knowledge of residents about the transmissibility and prevention of \\ tuberculosis: biopsychosocial implications \\ Análisis del conocimiento de moradores cuanto a la transmisibilidad y \\ prevención de la tuberculosis: implicaciones biopsicosocial \\ Patrick Leonardo Nogueira da Silva(1) \\ Maricy Kariny Soares Oliveira ${ }^{(2)}$ \\ Camilla Freitas Guimarães ${ }^{(2)}$ \\ Larissa Freitas Guimarães ${ }^{(2)}$ \\ Letícia Rosa Santos ${ }^{(3)}$ \\ Elaine Cristina Santos Alves ${ }^{(2)}$ \\ ${ }^{(1)}$ Fundação Hospitalar do Município de Espinosa - FHUMESP, Espinosa, MG, Brasil. \\ (2) Universidade Estadual de Montes Claros - UNIMONTES, Montes Claros, MG, Brasil. \\ ${ }^{(3)}$ Faculdades Integradas Pitágoras de Montes Claros - FIPMoC, Montes Claros, MG, Brasil.
}

Recebido: 23 fev 2017 Revisado: 11 de 2017 Aceito: 14 jan 2015

Autor de

correspondência:

Patrick Leonardo Nogueira da Silva

patrick_mocesp70@hotmail.com

Conflito de interesses: Os autores declaram não haver nenhum interesse profissional ou pessoal que possa gerar conflito de interesses em relação a este manuscrito.

\section{Resumo}

Os desastres ambientais são cada vez mais frequentes e causam grande impacto social, econômico e ambiental. Para que o cuidado ofertado aos expostos seja adequado e a recuperação integral, devem-se considerar as dimensões física, mental e social nas ações desenvolvidas. O presente estudo objetivou conhecer as possíveis atuações do psicólogo em situações de desastres ambientais e buscou descrever os impactos dos desastres sobre a saúde da população exposta a eles. Para tal, foi realizada uma revisão bibliográfica a partir de publicações feitas por instituições de referência e órgãos oficiais. No campo da Psicologia o debate sobre o assunto tem crescido, assim como a produção de conhecimento. Porém, ainda são escassas as publicações com orientações específicas para a atuação de psicólogos em situações de desastres ambientais. É consenso que as ações relacionadas aos desastres não devem restringir-se ao auxílio durante e após o ocorrido, sendo essenciais práticas preventivas que orientem e conscientizem a população para que sejam capazes de ajudar a minimizar o impacto dos eventos. 0 psicólogo em todas as etapas deve atuar para promover e proteger os direitos humanos, assim como auxiliar para que a população possa dar novo significado a experiência vivida. É necessário ampliar os debates e estruturar as pesquisas sobre o tema, para que se produzam orientações e diretrizes sólidas para uma assistência efetiva à população afetada

Descritores: Tuberculose; Efeitos psicossociais da Doença; Transmissão; Prevenção de Doenças. 


\begin{abstract}
Tuberculosis is considered an infectious disease, Mycobacterium tuberculosis is an etiological agent, in which it mainly affects the lungs and may develop in other organs of the body, and clinical manifestations are directly related to the affected organ. This study aimed to analyze residents' knowledge regarding the transmissibility and prevention of tuberculosis and its biopsychosocial implications. This is a descriptive, exploratory study with a quantitative approach, carried out with 118 clients of a Family Health Strategy of Minas Gerais. A structured questionnaire was used as a data collection instrument. Data were processed through non-parametric and non-probabilistic simple descriptive epidemiology. It was observed that the majority of the interviewees were female, aged 18-45 years, brown, with high school and had occupation. Most of the sample knows about the definition of the disease $(82.2 \%), 81.3 \%$ said that transmission occurs through the air, $88.1 \%$ believe that there are ways to prevent tuberculosis. Therefore, residents have satisfactory knowledge about the disease, but it is observed the persistence of paradigms in which they lead to social isolation and interfere in the individual/society dynamics.
\end{abstract}

Keywords: Tuberculosis; Cost of Illness; Transmission; Disease Prevention.

\title{
Resumen
}

La tuberculosis es considerada una enfermedad infecciosa, y Mycobacterium tuberculosis como el agente etiológico, que afecta principalmente a los pulmones y puede desarrollarse en otros órganos del cuerpo, y las manifestaciones clínicas están directamente relacionados con el órgano diana. Este estudio tuvo como objetivo analizar el conocimiento de los residentes sobre la transmisibilidad y la prevención de la tuberculosis y sus consecuencias biopsicosociales. Se trata de un estudio descriptivo, exploratorio con enfoque cuantitativo, realizado con 118 clientes una familia Estrategia de Salud de Minas Gerais. Se utilizó un cuestionario estructurado como un instrumento de recolección de datos. El análisis de datos se realiza a través de la epidemiología descriptiva sencilla no paramétrico y no probabilístico. Se observó que la mayoría de los encuestados eran mujeres, con edades entre 18-45 años, de color marrón con la escuela secundaria y tenía ocupación. La mayor parte de la muestra que se sabe acerca de la definición de la enfermedad (82,2\%), el 81,3\% dijo que la transmisión tiene lugar por vía aérea, el 88,1\% cree que hay maneras de prevenir la tuberculosis. Por lo tanto, los residentes tienen un conocimiento suficiente acerca de la enfermedad, sin embargo no es la persistencia de paradigmas en los cuales conducen al aislamiento social e interfieren con la dinámica individuo/sociedad.

Palabras-claves: Tuberculosis; Costo de Enfermedad; Transmisión; Prevención de Enfermedades.

\section{Introdução}

A Tuberculose - TBC é uma doença infectocontagiosa, causada por um microorganismo denominado Mycobacterium tuberculosis, que afeta principalmente os pulmões e pode se desenvolver em outros órgãos do corpo, como ossos, rins e meninges, sendo que as manifestações clínicas estão diretamente relacionadas com o órgão atingido. Esse bacilo se propaga por meio do ar, por meio de aerossóis expelidos por um doente com TBC pulmonar ao tossir, espirrar ou falar. A TBC é transmitida apenas em sua forma 
pulmonar e faríngea. A infecção ocorre quando os aerossóis contendo os bacilos expelidos são inalados por pessoas sadias, provocando a infecção tuberculosa e o risco de desenvolvimento e manifestação da doença. ${ }^{1}$

A TBC ainda é considerada um importante problema de saúde, em nível mundial, exigindo, assim, o desenvolvimento de estratégias para o seu controle, considerando tanto os aspectos humanitários, quanto econômicos e de saúde pública. ${ }^{2}$ A relevância da magnitude da TBC pode ser evidenciada nos dados epidemiológicos mundiais e nacionais. Em 2010 diagnosticou-se e notificou-se 6,2 milhões de casos de TBC, no mundo, sendo que 5,4 milhões se tratavam de casos novos, equivalentes a $65 \%$ dos casos estimados para o mesmo ano. O Brasil está entre os 22 países que concentram $82 \%$ dos casos de TBC no mundo. $^{3}$

Em 2011, foram notificados 69.245 casos de TBC no Brasil, com uma taxa de incidência para cada grupo de 100.000 habitantes de 45,2\% na região Norte, 35,9\% Nordeste, 37,6\% Sudeste, 33\% Sul, 22\% Centro-Oeste e 36\% no Brasil. Analisando a taxa de incidência da TBC no Brasil de 2001 (42,8\%) a 2011, observa-se uma queda significativa de 15,9 pontos. Entretanto, para avaliar as ações de controle da TBC é imprescindível que avalie também os percentuais de detecção de casos e percentual de cura dos mesmos. As metas recomendadas pela Organização Mundial de Saúde - OMS são: detectar 70\% e curar pelo menos $85 \%$ dos casos para que assim, os países comecem a reverter a situação da TBC em suas localidades. O Brasil detectou $88 \%$ dos casos estimados pela OMS para 0 ano de 2010; porém, o alcance do percentual recomendado pela OMS para o indicador de cura ainda é um desafio para o país, não atingindo, portanto, a meta estabelecida. ${ }^{4}$

No que diz respeito à prevenção e o controle da doença, estes estão diretamente relacionados com a Vigilância Epidemiológica dos casos confirmados e seus comunicantes, incluindo o acesso ao tratamento medicamentoso, a disponibilidade de suporte laboratorial para a pesquisa de casos novos e a produção de informações que permitam a caracterização da doença e o monitoramento dos casos suspeitos e confirmados. Para 
tanto, a manutenção periódica, atualização e avaliação da base de dados do Sistema de Informação de Agravos e Notificações - SINAN são condições fundamentais para o acompanhamento da situação epidemiológica dos casos de TBC. 5 ,6 Pesquisas realizadas em diversas regiões do país permitem identificar o perfil dos indivíduos acometidos pela TBC.

Em estudo realizado na região central de São Paulo, SP, 69\% dos investigados corresponderam a indivíduos do sexo masculino, com média de idade de 40 anos. E no que tange à escolaridade, cerca de $70 \%$ dos usuários estudados cursaram pelo menos um ano escolar, e apenas $9 \%$ completaram 12 anos ou mais de escolaridade. ${ }^{7}$ Com a aquisição da TBC, os portadores desta doença sofrem, não só pelas manifestações clínicas, como também pela possibilidade de vivenciar as consequências das repercussões biopsicossociais que a TBC pode ocasionar, atingindo consequentemente as relações sociais em geral. Neste sentido, a doença faz com que a pessoa seja identificada como perigo à saúde das demais. Na realidade, o que ela teme consiste na rejeição, afastamento dos familiares e o próprio temor da população em geral. ${ }^{8}$

O próprio tratamento traz vivências negativas aos indivíduos com TBC. A insegurança, a preocupação e o desconhecimento geram medo, e consequentemente sofrimento. Este sofrimento pode ser decorrente do medo da transmissão, medo do preconceito e medo da morte. O Medo da transmissão, por sua vez, é marcado por sofrimento antecipatório em transmitir a doença para outras pessoas, principalmente para os membros familiares, podendo, dessa forma, contribuir para seu isolamento social, mesmo depois de serem avisados de que não representavam riscos para a família. ${ }^{9}$ No que diz respeito ao medo do preconceito, muitas vezes esse pode originar também da própria pessoa portadora da TBC, podendo variar de acordo com suas crenças e concepções acerca da doença, e novamente influenciando diretamente em seus relacionamentos sociais. $^{8}$

O medo da morte, por sua vez, é decorrente do estigma da TBC, visto que, 
antigamente não havia tratamento para a doença. ${ }^{9}$ Além disso, fatores de ordem sociocultural podem dificultar o tratamento, como o baixo nível de escolaridade, desconhecimento sobre a TBC e a não aceitação da doença, fazendo necessário, para isto, que atenção especial seja dada aos usuários com menor grau de escolaridade. ${ }^{6}$

Esta pesquisa justifica-se pela relevância de estudar a TBC, devido à necessidade de investigar suas repercussões, bem como mitos, tabus e paradigmas além de conhecer a real situação da comunidade no que se refere à informação sobre o mecanismo de transmissão e prevenção da doença. Portanto, objetivou analisar o conhecimento de moradores quanto à transmissibilidade e prevenção da TBC e suas implicações biopsicossociais.

\section{Metodologia}

Trata-se de um estudo descritivo, exploratório, com abordagem quantitativa, realizado com 118 moradores cadastrados na Estratégia Saúde da Família - ESF do bairro Nossa Senhora de Fátima do município de Montes Claros, Minas Gerais - MG, Brasil. Foi enviado à Secretaria Municipal de Saúde - SMS de Montes Claros, MG, uma carta de apresentação das diretrizes da pesquisa e um Termo de Concordância Institucional - TCI na qual o mesmo foi assinado de modo a autorizar a realização do estudo.

Foram adotados os seguintes critérios de inclusão para participação na pesquisa: clientes com idade superior a 18 anos; e ser residente da área de abrangência da ESF. Utilizou-se um questionário estruturado como instrumento de coleta de dados, contendo 20 questões objetivas. Os dados foram coletados durante o mês de maio de 2011.

A amostra foi selecionada por acessibilidade. Após a coleta, os dados foram armazenados no banco de dados StatisticalPackage for the Social Sciences- SPSS e tabulados por meio do programa Microsoft Exce ${ }^{\circledR}$ for Windows $X P^{\circledR}$, versão 2010, e representado estatisticamente em tabelas por meio de frequências absolutas (n), percentuais (\%) e medidas de tendência central (MTC), tal como a média aritmética 
ponderada (MAP) e o desvio padrão (DP). O tratamento dos dados se deu através de epidemiologia descritiva simples não paramétrica e não probabilística.

Este estudo obedeceu aos preceitos éticos estabelecidos pela Resolução nº 196/96 do Conselho Nacional de Saúde - CNS, revogada pela Resolução n 466/2012, ${ }^{10}$ na qual regulamenta a pesquisa envolvendo seres humanos. O projeto de pesquisa foi apreciado e aprovado pelo Comitê de Ética em Pesquisa da Universidade Estadual de Montes Claros (CEP UNIMONTES), sob parecer consubstanciado n 1993/2010. Os participantes foram devidamente orientados quanto às diretrizes do estudo na qual assinaram o Termo de Consentimento Livre e Esclarecido - TCLE para participação e autorização do mesmo.

\section{Resultados}

Quanto ao perfil socioeconômico e demográfico dos participantes, observou-se a prevalência do sexo feminino $(68,6 \%)$ composto por adultos jovens com faixa etária entre 18-45 anos $(73,8 \%)$ e média de idade de $37,14 \pm 15,2028$ anos, raça parda (53,5\%), Ensino Médio (47,2\%) e apresentam ocupação (58,5\%) (Tabela 1).

Tabela 1. Perfil socioeconômico e demográfico de Montes Claros, 2011.

\begin{tabular}{|c|c|c|c|}
\hline Variáveis & $\mathbf{n}$ & $\%$ & $M A P \pm D P$ \\
\hline \multicolumn{4}{|l|}{ Sexo } \\
\hline Masculino & 37 & 31,4 & - \\
\hline Feminino & 81 & 68,6 & - \\
\hline \multicolumn{4}{|l|}{ Faixa etária (anos) } \\
\hline $18-45$ & 87 & 73,8 & \multirow{2}{*}{$37,14 \pm 15,2028$} \\
\hline $46-60$ & 31 & 26,2 & \\
\hline \multicolumn{4}{|l|}{ Cor/Raça } \\
\hline Parda & 63 & 53,5 & - \\
\hline Negro & 20 & 17,1 & - \\
\hline Branco & 35 & 29,4 & - \\
\hline \multicolumn{4}{|l|}{ Escolaridade } \\
\hline Ensino básico & 15 & 12,6 & - \\
\hline Ensino fundamental & 39 & 33,2 & - \\
\hline Ensino médio & 56 & 47,2 & - \\
\hline
\end{tabular}


Ensino superior

\section{Ocupação}

Sim

$69 \quad 58,5$

Não

Fonte: Elaborado pelos autores (2017).

Nota: $n=118$.

Sobre o conhecimento da TBC, $82,2 \%$ dos entrevistados afirmaram conhecer sobre a doença, bem como o seu órgão-alvo, sendo este o pulmão, mas também podem atingir outros órgãos, sendo esta contagiosa e transmitida por via aérea. Em contrapartida, 10,3\% apenas relataram não saber. A falta de conhecimento da população sobre a TBC ainda é um dos principais desafios enfrentados para o controle da doença, de forma a levar ao estigma e preconceito da sociedade (Tabela 2).

Tabela 2. Perfil biopsicossocial do conhecimento dos participantes quanto à TBC, sua transmissibilidade e prevenção de Montes Claros, 2011.

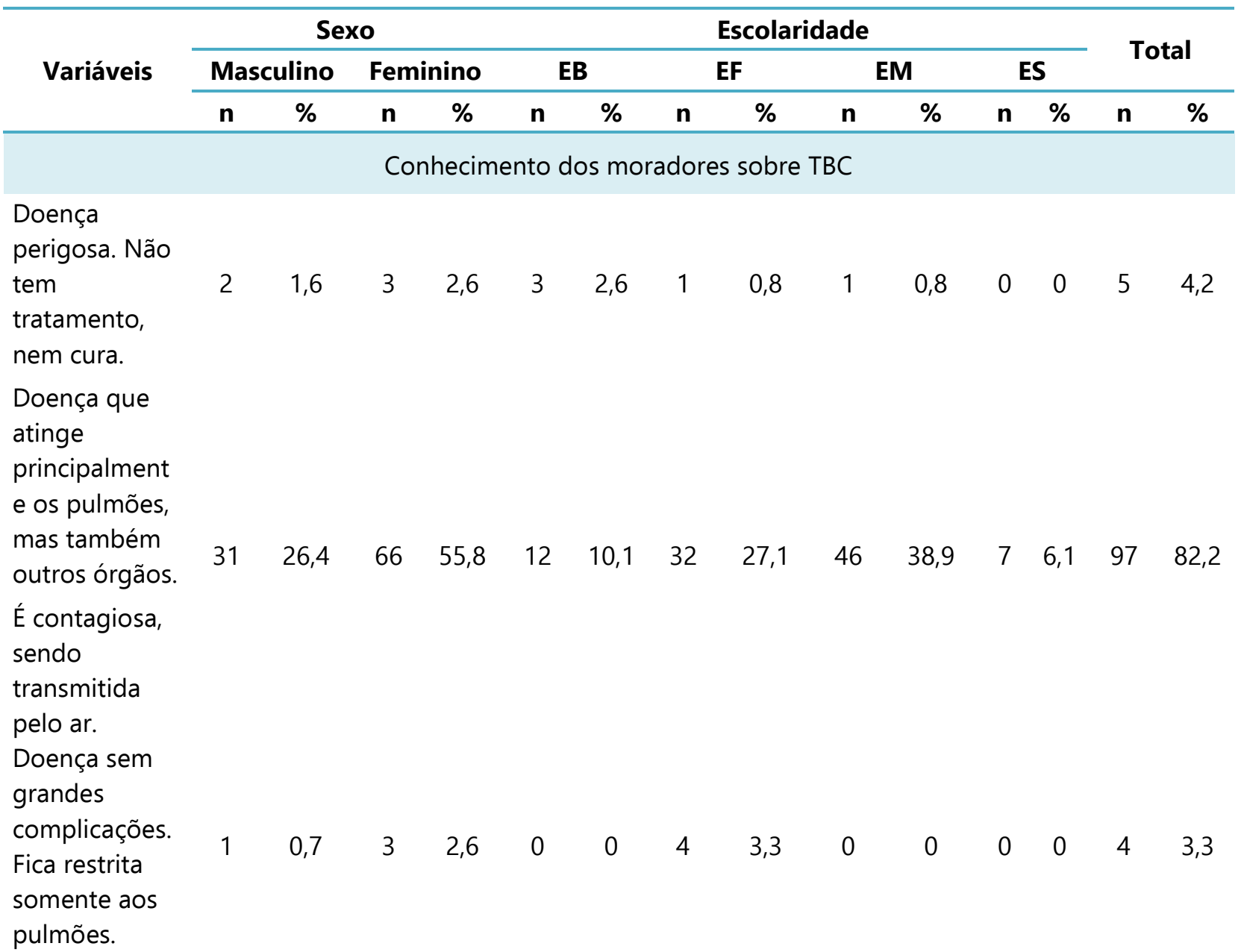


$\begin{array}{lllllllllllllll}\text { Não sabe. } & 3 & 2,6 & 9 & 7,7 & 0 & 0 & 2 & 1,8 & 9 & 7,7 & 1 & 0,8 & 12 & 10,3\end{array}$

Total $\begin{array}{llllllllllllll}37 & 31,3 & 81 & 68,7 & 15 & 12,7 & 39 & 33 & 56 & 47,4 & 8 & 6,9 & 118 & 100\end{array}$

Informação quanto à via de transmissão da TBC

\begin{tabular}{|c|c|c|c|c|c|c|c|c|c|c|c|c|c|c|}
\hline Água. & 1 & 0,8 & 0 & 0 & 1 & 0,8 & 0 & 0 & 0 & 0 & 0 & 0 & 1 & 0,8 \\
\hline $\begin{array}{l}\text { Picada de } \\
\text { mosquito. }\end{array}$ & 2 & 1,6 & 3 & 2,6 & 0 & 0 & 2 & 1,7 & 3 & 2,5 & 0 & 0 & 5 & 4,2 \\
\hline Ar. & 30 & 25,4 & 66 & 55,9 & 12 & 10,1 & 32 & 27,1 & 45 & 38,1 & 7 & 6 & 96 & 81,3 \\
\hline Outras vias. & 4 & 3,3 & 12 & 10,4 & 2 & 1,8 & 5 & 4,2 & 8 & 6,8 & 1 & 0,9 & 16 & 13,7 \\
\hline Total & 37 & 31,3 & 81 & 68,7 & 15 & 12,7 & 39 & 33 & 56 & 47,4 & 8 & 6,9 & 118 & 100 \\
\hline \multicolumn{15}{|c|}{ Informação quanto à existência de prevenção da TBC } \\
\hline Sim & 32 & 27,1 & 72 & 61 & 13 & 11 & 34 & 28,8 & 49 & 41,4 & 8 & 6,9 & 104 & 88,1 \\
\hline Não & 5 & 4,2 & 9 & 7.7 & 2 & 1,7 & 5 & 4,2 & 7 & 6 & 0 & 0 & 14 & 11,9 \\
\hline Total & 37 & 31,3 & 81 & 68,7 & 15 & 12,7 & 39 & 33 & 56 & 47,4 & 8 & 6,9 & 118 & 100 \\
\hline
\end{tabular}

Não se

aproximaria

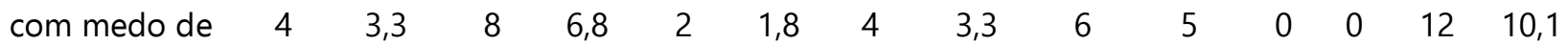
contrair a

doença.

Não

conversaria

$\begin{array}{lllllllllllllll}\text { com medo de } & 0 & 0 & 2 & 1,6 & 0 & 0 & 1 & 0,8 & 1 & 0,8 & 0 & 0 & 2 & 1,6\end{array}$

contrair a

doença.

Aproximaria e

conversaria

normalmente,

$\begin{array}{llllllllllllll}20 & 16,9 & 43 & 36,4 & 8 & 6,7 & 21 & 17,7 & 30 & 25,4 & 4 & 3,5 & 63 & 53,3\end{array}$

sem medo.

Aproximaria e

conversaria,

mas com

$\begin{array}{llllllllllllll}13 & 11 & 28 & 24 & 5 & 4,2 & 13 & 11,2 & 19 & 16,2 & 4 & 3,4 & 41 & 35\end{array}$

medo.

Total

$\begin{array}{llllllllllllll}37 & 31,3 & 81 & 68,7 & 15 & 12,7 & 39 & 33 & 56 & 47,4 & 8 & 6,9 & 118 & 100\end{array}$ Percepção quanto à interferência da TBC na vida do portador

\begin{tabular}{lcccccccccccccc} 
Sim & 28 & 23,7 & 61 & 51,7 & 11 & 9,3 & 29 & 24,5 & 42 & 35,5 & 7 & 6,1 & 89 & 75,4 \\
Não & 9 & 7,6 & 20 & 17 & 4 & 3,4 & 10 & 8,5 & 14 & 11,9 & 1 & 0,8 & 29 & 24,6 \\
Total & $\mathbf{3 7}$ & $\mathbf{3 1 , 3}$ & $\mathbf{8 1}$ & $\mathbf{6 8 , 7}$ & $\mathbf{1 5}$ & $\mathbf{1 2 , 7}$ & $\mathbf{3 9}$ & $\mathbf{3 3}$ & $\mathbf{5 6}$ & $\mathbf{4 7 , 4}$ & $\mathbf{8}$ & $\mathbf{6 , 9}$ & $\mathbf{1 1 8}$ & $\mathbf{1 0 0}$ \\
\hline
\end{tabular}

Fonte: Elaborado pelos autores (2017).

Nota: $\mathrm{n}=118 ; \mathrm{EB}=$ Ensino Básico; $\mathrm{EF}$ = Ensino Fundamental; $\mathrm{EM}=$ Ensino Médio; $\mathrm{ES}$ = Ensino Superior.

De acordo à mesma Tabela 2, nota-se que $81,3 \%$ das pessoas relataram que a transmissão da TBC se dá pelo ar, o que demonstra concordância com a literatura. Verifica- 
se, no entanto, que há um número considerável de participantes da pesquisa que não sabia o modo de transmissão da doença, uma vez que $13,7 \%$ responderam a opção "Outras vias". Observa-se que a maioria dos entrevistados $(88,1 \%)$ afirmou haver prevenção para a TBC. Ao se deparar com um portador de TBC, constatou-se que 53\% dos entrevistados não têm medo de contrair a doença, porém observa-se que 34,7\% apresentam receio de se aproximar do portador de TBC.

A leitura da Tabela 2 ainda esclarece que 75,4\% da amostra acreditam que um portador de TBC pode ter uma vida normal, enquanto que $24,6 \%$ responderam que a doença afeta a vida diária. É verificado que a população não sabe quais as repercussões que a TBC implica na vida do doente, uma vez que estes sofrem com a aquisição desta doença, não só sintomas da doença, como também por vivenciar preconceito e rejeição, o que atinge as relações sociais.

\section{Discussão}

Estudos têm apontado uma maior proporção de casos de TBC em homens, em relação às mulheres, bem como, maior prevalência de baixa escolaridade nesses usuários. ${ }^{6,7}$ Estudos recentes sugerem que a modificação nas estimativas nacionais de incidência da TBC está mais associada às mudanças nos índices socioeconômicos e condições gerais de saúde da população do que ao desempenho de programas de controle desses agravos. $^{11}$

Em pesquisa realizada em Londrina, Paraná, $P R$, observou-se maior prevalência da TBC em indivíduos do sexo masculino (68\%) com faixa etária mais acometida variando entre 41 e 60 anos. E, no que se refere ao grau de escolaridade, 22,4\% dos usuários estudados tinham entre quatro e sete anos de estudo, sendo este tempo também observado entre os que abandonaram o tratamento $(33,3 \%) .{ }^{6}$ Os dados acima reforçam a necessidade de se investir na informação e educação da população. 
Outros estudos apontaram associação positiva para as seguintes variáveis: sexo (masculino), faixa etária (30 a 54 anos), analfabetismo, imigração, posse de poucos bens de consumo, baixa renda ou rendimento afixo, histórico prisional, alcoolismo, estado civil (separado, viúvo ou solteiro), carência alimentar e contato prévio com paciente de TBC e ainda relacionam estas variáveis com adesão e ao início mais precoce do tratamento. ${ }^{11,12}$ Os indicadores socioeconômicos referentes aos baixos níveis de renda e escolaridade poderiam aumentar a vulnerabilidade à TBC ao refletir o acesso individual e desigual à informação, a benefícios oriundos do conhecimento, aos bens de consumo e ao serviço de saúde. ${ }^{11}$ Corroborando com a necessidade de estabelecer estratégias com as populações mais susceptíveis como os entrevistados deste estudo (idade 18 a 45 anos, pouca escolaridade e baixa renda).

Não foi encontrado consenso entre os estudos pesquisados relacionando maior conhecimento ao sexo feminino, porém estudos sobre conhecimento para outras patologias mostraram que o sexo feminino e a escolaridade foram importantes para um maior conhecimento sobre doenças como o câncer e as doenças sexualmente transmissíveis (DST).

Além disso, no que diz respeito ao tratamento, a maioria da população que não aderiu ao tratamento, era composta por pacientes mais jovens, especialmente homens, fato que pode estar atribuído ao estilo de vida desta população que normalmente faz uso de bebidas alcoólicas e possui horários irregulares para a alimentação, o que contribui para a interrupção do tratamento. ${ }^{6}$ Embora a não adesão esteja relacionada a vários fatores, sobretudo os demográficos, essa ocorre mais frequentemente em indivíduos do sexo masculino e baixa escolaridade. ${ }^{13}$

O estigma vinculado à falta de conhecimento é visto como a maior barreira ao acesso precoce aos serviços de saúde. Confirmar o diagnóstico implica ter sua identidade alterada em função de uma doença vinculada por estigma e incapacidades. Dessa forma, os usuários tendem a retardar a busca pelo atendimento, bem como a realização de 
exames diagnósticos e, assim, apresentam dificuldades em aderir ao tratamento. Sendo assim, estes fatores interferem na cura do doente ou agravamento do seu quadro. ${ }^{14}$

Dessa forma, a educação em saúde torna-se um requisito essencial na promoção do cuidado às pessoas com TBC, contribuindo para o combate do estigma relacionada a essa enfermidade, uma vez que conhecer a doença permite um comportamento adequado diante de situações delicadas e impede que mitos e informações inverídicas continuem a interferir no bom andamento do tratamento. ${ }^{15}$ Nesse sentido, é de suma importância que o serviço de saúde esclareça à comunidade quanto aos aspectos importantes da doença, como sua transmissão, prevenção e tratamento. ${ }^{1}$

Quanto à transmissibilidade do bacilo, a fala, o espirro e, principalmente, a tosse de um doente com TBC, são as principais formas de disseminação já que lançam no ar partículas pequenas de aerossóis contaminados. A transmissão geralmente ocorre em locais fechados, nos quais as partículas expelidas pelo doente podem permanecer no ar. Mas, a ventilação constante e luz solar direta removem as partículas e matam os bacilos rapidamente. ${ }^{1}$ Assim sendo, diante da prevalência da TBC no Brasil, evidencia-se ainda grande desconhecimento da população acerca da mesma, de forma a fazer-se necessário uma maior divulgação sobre o conceito, bem como suas formas de transmissão e manifestações iniciais, de forma a promover sua prevenção e diagnóstico precoce. ${ }^{8}$

O melhor recurso de prevenção da TBC continua sendo a detecção precoce dos casos existentes e o seu tratamento correto, visto que os indivíduos doentes constituem a maior fonte de infecção. ${ }^{2}$ O Bacillus Calmette-Guérin, mais conhecido como BCG, é a vacina que protege contra as manifestações graves da primo-infecção, como as disseminações hematogênicas e a meningoencefalite, mas não evita a infecção tuberculosa. A proteção se mantém por 10 a 15 anos, não protege os indivíduos já infectados, por isso faz-se necessário que vacinem crianças quanto antes possível, após o nascimento, sendo indicada para as crianças de zero a quatro anos de idade, sendo obrigatória para menores de um ano. ${ }^{1}$ 
A quimioprofilaxia com isoniazida também constitui uma forma de prevenção, devendo essa ser administrada em pessoas infectadas pelo M. tuberculosis, diminuindo assim o risco de desenvolvimento da doença. ${ }^{1}$ Comunicante é definido como toda pessoa que convive no mesmo ambiente com o caso-índice, no momento do diagnóstico da TBC, também merecem atenção especial quanto à quimioprofilaxia. Todos devem ser avaliados clinicamente, de forma criteriosa, a fim de definir corretamente pelo início ou não da quimioprofilaxia. $^{16}$

A falta de informação faz com que "medos" em relação à doença surjam, o que é um grande problema, pois o desenvolvimento dessa patologia não depende de um único fator, a presença do bacilo, mas de uma soma deles, tal como os fatores ambientais, o tipo e continuidade do contato e a forma de apresentação da doença do caso-índice. ${ }^{2} \mathrm{~A}$ TBC ainda é uma doença cercada de preconceito pela sociedade e também pelo próprio paciente. Muitas vezes, a população carrega uma bagagem de informações errôneas, especialmente quanto ao modo de transmissão, evolução e contágio da TBC, que consequentemente acaba por gerar um comportamento de isolamento social. ${ }^{17}$

Diante do afastamento e medo da sociedade em contrair a TBC, há sentimentos e emoções negativos do indivíduo portador. Devido o isolamento social que vivenciam, dificuldades em realizarem o tratamento, medo do contágio e pela mudança na percepção da imagem corporal, o adoecer por TBC é percebido como sofrimento, expressado como perdas, tristeza, descontentamento e revolta. ${ }^{8}$ A TBC é uma doença contagiosa e, em geral, as doenças que são contagiosas trazem um estigma muito grande para a pessoa que é portadora da doença. Mas existe todo um movimento da sociedade para integrar essas pessoas porque não existe uma razão para se marginalizar. As doenças contagiosas hoje têm tratamento e a TBC é uma doença que tem cura em praticamente $100 \%$ dos casos com tratamento correto e bem conduzido. ${ }^{1}$

Apesar de se tratar de uma enfermidade que tem cura, o estigma ainda está presente nos dias atuais. Nota-se ainda, uma mescla de representações da doença 
associadas aos determinantes sociais da mesma, persistindo o imaginário social do passado quanto ao perfil do paciente e da própria a doença. ${ }^{18}$

\section{Conclusão}

A análise do conhecimento de moradores quanto à transmissibilidade e prevenção da TBC, suas implicações biopsicossociais, permitiu concluir que a população estudadaafirma ter conhecimento acerca do conceito da TBC e suas formas de transmissão, porém ainda persiste a desinformação sobre o real conceito da doença, bem como sua forma de transmissão e prevenção. Acirra-se a esta discussão, o perfil demográfico dos entrevistados e seus indicadores socioeconômicos referentes a baixos níveis de renda e escolaridade como potenciais para aumentar a vulnerabilidade à TBC devido ao acesso individual e desigual aos meios de informação e benefícios oriundos do conhecimento. Apesar das informações sobre saúde terem cada vez maior destaque na mídia e demais meios de comunicação, observa-se a persistência de paradigmas na qual levam ao isolamento social e interferem na dinâmica indivíduo/sociedade.

Dessa forma faz-se necessário desenvolver estratégias de educação que realmente sejam eficazes e conduza o serviço da Atenção Primária a Saúde - APS, como a operacionalização de práticas de educação da comunidade em relação às doenças infectocontagiosas, especialmente a $\operatorname{TBC}$ e, aprimoramento do trabalho de prevenção baseado em diretrizes pedagógicas com metodologias de inclusão da comunidade no constante combate à desinformação, estigmatização sobre a TBC e suas repercussões sociais, abordando tais atores nas escolas, associações comunitárias, segmentos esportivos e religiosos, desenvolvendo cidadania por meio de práticas de saúde emancipatórias.

\section{Referências}

1. Brasil. Ministério da Saúde. Secretaria de Atenção à Saúde. Departamento de Atenção Básica. Vigilância em saúde - dengue, esquistossomose, hanseníase, malária, tracoma e tuberculose. 2a ed. Brasília, DF: MS; 2008. 
2. Brasil. Ministério da Saúde. Grupo Hospitalar Conceição. Centro Federal de Ensino e Pesquisa em Saúde. Serviço de Saúde Comunitária. Tuberculose na Atenção Primária à Saúde. Brasília, DF: 2011.

3. World Health Organization. Global tuberculosis control: WHO Report 2010. Geneva: WHO; 2010.

4. Brasil. Ministério da Saúde. Secretaria de Vigilância em Saúde. Boletim epidemiológico especial tuberculose. Brasília, DF: MS; 2012.

5. Nogueira JA, Sá LD, França UM, Almeida SA, Lima DS, Figueiredo TMRM, et al. O sistema de informação e o controle da tuberculose nos municípios prioritários da Paraíba Brasil. Rev Esc Enferm USP. 2009;43(1):125-31.

6. Giroti SKO, Belei RA, Moreno FN, Silva FS. Perfil dos pacientes com tuberculose e os fatores associados ao abandono do tratamento. Cogitare Enferm. 2010;15(2):271-7. http://dx.doi.org/10.5380/ce.v15i2.17860.

7. Caliari JS, Figueiredo RM. Tuberculose: perfil de doentes, fluxo de atendimento e opinião de enfermeiros. Acta Paul Enferm. 2012;25(1):43-7.

http://dx.doi.org/10.1590/S0103-21002012000100008.

8. Souza SS, Silva DMGV, Meirelles BHS. Representações sociais sobre a tuberculose. Acta Paul Enferm. 2010;23(1):23-8. http://dx.doi.org/10.1590/S0103-21002010000100004.

9. Dias AAL. Vivências psicossociais de pacientes que concluíram o tratamento de tuberculose no interior do Estado de São Paulo: um estudo clínico-qualitativo [dissertação]. São Carlos: Universidade Federal de São Carlos; 2011. 95 f.

10. Brasil. Ministério da Saúde. Conselho Nacional de Ética em Pesquisa. Resolução N 466, de 12 de dezembro de 2012. A presente Resolução incorpora, sob a ótica do indivíduo e das coletividades, referenciais da bioética, tais como, autonomia, não maleficência, beneficência, justiça e equidade, dentre outros, e visa a assegurar os direitos e deveres que dizem respeito aos participantes da pesquisa, à comunidade científica e ao Estado. Projetos de pesquisa envolvendo seres humanos deverão atender a esta Resolução. Brasília, DF: CONEP; 2012.

11. San Pedro A, Oliveira RM. Tuberculose e indicadores socioeconômicos: revisão sistemática da literatura. Rev Panam Salud Publica. 2013;33(4):294-30.

12. Ximenes RAA, Albuquerque MFPM, Souza WV, Montarroyos UR, Diniz GT, Luna CF, et al. Is it better to be rich in a poor area or poor in a rich area? A multilevel analysis of a 
case-control study of social determinants of tuberculosis. Int J Epidemiol. 2009;38(5):1285-96. http://dx.doi.org/10.1093/ije/dyp224.

13. Chirinos NEC, Meirelles BHS. Fatores associados ao abandono do tratamento da tuberculose: uma revisão integrativa. Texto Context Enferm. 2011;20(3):599-606.

14. Sá LD, Barreto AJR, Nogueira JA, Cunha FTS, Palha PF, Villa TCS. A discursividade de gestores sobre aspectos relacionados ao retardo do diagnóstico de tuberculose. Rev Esc Enferm USP. 2013;47(5):1170-7. http://dx.doi.org/10.1590/S0080-623420130000500022.

15. Sá LD, Gomes ALC, Nogueira JA, Villa TCS, Souza KMJ, Palha PF. Intersetorialidade e vínculo no controle da tuberculose na Saúde da Família. Rev Latino-Am Enferm. 2011;19(2):387-95.

16. Brasil. Ministério da Saúde. Secretaria de Vigilância em Saúde. Departamento de Vigilância Epidemiológica. Manual de recomendações para o controle da tuberculose no Brasil. Brasília, DF: 2011.

17. Assunção CG, Seabra JDR, Figueiredo RM. Percepção do paciente com tuberculose sobre a internação em hospital especializado. Cienc Enferm. 2009;15(2):69-77. http://dx.doi.org/10.4067/S0717-95532009000200008.

18. Wendling APB, Modena CM, Schall VT. Tuberculose: o estigma na visão de gerentes de centros de saúde de Belo Horizonte, MG - Brasil. Cad ESP. 2010; 4(2): 11-8. 


\section{Minicurrículo}

Patrick Leonardo Nogueira da Silva | ORCiD: 0000-0003-2399-9526

Especialista em Enfermagem do Trabalho pela Faculdade de Guanambi - FG. Enfermeiro da Fundação Hospitalar do Município de Espinosa - FHUMESP. Professor da Universidade Estadual de Montes Claros UNIMONTES.

Maricy Kariny Soares Oliveira | ORCiD: 0000-0002-1303-8513

Doutora em Ciências da Educação pela Universidade Evangelista do Paraguai e Cambridge - UEPC. Professora do Departamento de Enfermagem da Universidade Estadual de Montes Claros - UNIMONTES.

Camilla Freitas Guimarães | ORCiD: 0000-0002-5658-3263

Enfermeira pela Universidade Estadual de Montes Claros - UNIMONTES.

Larissa Freitas Guimarães | ORCiD: 0000-0001-8511-5269

Especialista em Saúde da Família na modalidade Residência Multiprofissional da Universidade Estadual de Montes Claros - UNIMONTES. Enfermeira pela UNIMONTES.

Letícia Rosa Santos | ORCID: 0000-0001-5684-7081

Especialista em Gestão e Auditoria em Processos de Saúde pelo Instituto de Ensino e Pesquisa da Santa Casa de Belo Horizonte - IEP-SCBH. Enfermeira pela Faculdades Integradas Pitágoras de Montes Claros - FIPMoc.

Elaine Cristina Santos Alves | ORCID: 0000-0003-1357-9341

Mestre em Ciências da Saúde pela Universidade Federal de São Paulo - UNIFESP. Professora do Departamento de Enfermagem da Universidade Estadual de Montes Claros - UNIMONTES. 